\title{
New Results from High Energy Gamma-Ray Astronomy
}

\author{
H. J. Völk \\ Max-Planck-Institut für Kernphysik, P. O. Box, 69029 Heidelberg, Germany \\ email: Heinrich.Voelk@mpi-hd.mpg.de
}

\begin{abstract}
High energy gamma-ray astronomy has recently made significant progresss through ground-based instruments like the H.E.S.S. array of imaging atmospheric Cherenkov telescopes. The unprecedented angular resolution and the large field of view has allowed to spatially resolve for the first time the morphology of gamma-ray sources in the $\mathrm{TeV}$ energy range. The experimental technique is described and the types of sources detected and still expected are discussed. Selected results include objects as different as a Galactic binary Pulsar, the Galactic Center and Supernova Remnants but they also concern the diffuse extragalactic optical/infrared radiation field. Finally, a scan of the Galactic plane in $\mathrm{TeV}$ gamma rays is described which has led to a significant number of new $\mathrm{TeV}$ sources, many of which are still unidentified in other wavelengths. The field has a close connection with X-ray astronomy which allows the study of the synchrotron emission from these very high energy sources.
\end{abstract}

Keywords. radiation mechanisms: nonthermal, pulsars, Galaxy: center, acceleration of particles, supernova remnants, gamma rays: observations, cosmology: observations.

\section{Imaging Air Cherenkov Telescopes and the H.E.S.S. experiment}

The principles of the Air Cherenkov Technique were invented in the 1950ies, with a first astronomical success in the late 1980ies, when the Whipple telescope in Arizona detected the Crab Nebula (Weekes et al. (1989)).

The recent progress in VHE $\gamma$-ray astronomy with the H.E.S.S. experiment in Namibia was possible through several new developments in the 1990ies (i) the stereoscopic technique, pioneered by the German/Spanish/Armenian HEGRA experiment on La Palma (ii) the development of fine pixel "cameras" as focal plane detectors ( $C A T$ telescope in the French Pyrenees) (iii) the realization of the spatial extension of most of the expected $\gamma$-ray sources and thus the necessity for wide field-of-view (FoV) detectors allowing also sky surveys, and (iv) the need for the detection of "many", spatially extended nearby sources to solve the problem of Cosmic Ray origin in our Galaxy. It is therefore advantageous to observe the Galactic disk in the southern sky which led to the choice of the Gamsberg area in Namibia in Southern Africa.

Fig. 1a shows two of the four telescopes of the High Energy Stereoscopic System (H.E.S.S.) at 1800 m.a.s.l. It is operated by a large collaboration led by the Max Planck Institute for Nuclear Physics in Heidelberg. The four telescopes operate in coincidence (for more technical details, see the Web page http://www.mpi-hd.mpg.de/hfm/ HESS/HESS/html).

Overall the resulting H.E.S.S. sensitivity (4 telescopes) is as follows: 1 hour of observation time for a detection of an energy flux density of $10^{-11}\left(10^{-12}\right) \mathrm{erg} \mathrm{cm}^{-2} \mathrm{~s}^{-1}$ at $100 \mathrm{GeV}(1 \mathrm{TeV})$. With this performance the Crab Nebula can be detected at Zenith in $\sim 30$ s. For comparison, the 1989 detection required $\sim 50 \mathrm{hr}$. 

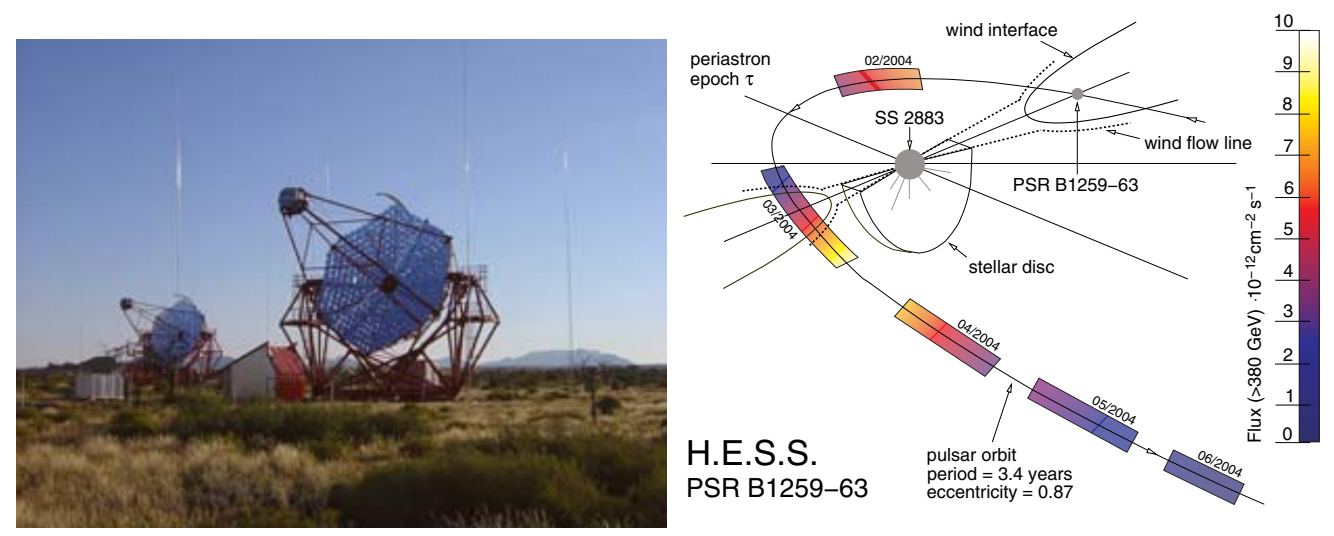

Figure 1. (Left:) The H.E.S.S. experiment in Namibia, near the famous Gamsberg. Two of the four 13m-telescopes are shown with their "cameras" in the focal point. (Right:) Orbital scheme of PSR B1259-63 with respect to the line of sight. The Pulsar approaches the stellar wind equatorial plane prior to periastron "behind" it.

\section{Science with high energy gamma-ray astronomy}

The two main fields of very high energy (VHE) $\gamma$-ray astronomy are High Energy Astrophysics and Observational Cosmology.

\subsection{High Energy Astrophysics}

High Energy Astrophysics concerns the most energetic and violent processes in the Universe, and in particular their nonthermal aspects. We have to expect that the nonthermal energy content $U_{\text {nonth }}$ of relativistic baryonic particles is in most regions of the Universe comparable to the energy densities in the thermal gas $U_{t h}$ and the magnetic fields $U_{\text {mag }}$, i.e. $U_{\text {nonth }} \sim U_{t h} \sim U_{\text {mag }}$. This should at least be true "everywhere" in galaxies and clusters of galaxies. But it holds probably also beyond, wherever cosmic structure formation with its violent, supersonic flows of baryonic matter has taken place or is still occurring. As a result of interactions of individual particles with collective excitations of the system, the particle sources and the associated nonthermal radiation should be characterized by power-law energy spectra rather than by thermal Maxwellian distributions

Because of its expected ubiquity I call this component the "Nonthermal Universe". Its study is intimately connected with that of stellar explosions, rapid outflows from galaxies, energy losses of extreme compact objects, and high-energy accretion processes up to the very largest spatial scales.

The types of VHE $\gamma$-ray sources found/expected in the Galaxy are Pulsar Nebulae, Supernova Remnants, X-ray Binaries ("Micro-Quasars"), Diffuse Galactic emission, Molecular Clouds, or possibly new source types. Extragalactic sources are Active Galactic Nuclei (e.g. Blazars), Radio Galaxies, Gamma-ray Pair Halos, Starburst Galaxies, Galaxy Mergers, Clusters of Galaxies, and Gamma-Ray Bursts.

\subsection{Observational Cosmology}

A major aspect of cosmology is cosmic structure formation. One of its consequences is the Extragalactic Background Light (EBL), the thermal, diffuse optical/infrared extragalactic background radiation from stars and Black Holes in galaxies and its re-radiation in the infrared. The EBL informs us about the epochs of galaxy formation and the history of their evolution. 
$\mathrm{TeV} \gamma$-quanta are absorbed by pair production on these low energy photons and therefore the spectra of distant extragalactic VHE $\gamma$-ray sources are expected to exhibit characteristic absorption features. They are the result of the magnitude and spectral variation of this background. Whereas a direct astronomical measurement of the EBL is very difficult, the $\gamma \gamma$-absorption is free of such complications. Its measurement for objects at different redshifts $z$ should in principle allow even the resolution of the EBL in $z$. Very recently, measurements of two different Blazars with known redshift that were newly discovered by the H.E.S.S. Collaboration have indeed been used (Aharonian et al. (2005a); see also the talk by J. Quinn in these Proceedings) to give the most stringent upper limit on the EBL in the optical/near-infrared band to date. It appears significantly lower than expected from the current "direct" estimates and very close to the absolute lower limit represented by the integrated light of resolved galaxies (Madau \& Pozetti (2000)). Apart from resolving the EBL largely into individual galaxies, this upper limit is especially in conflict with the claims of a high EBL flux at near-infrared wavelengths (Matsumoto et al. (2005); Cambresy et al. (2001)) which was often envisaged to be the result of radiation massively produced in the early Universe $(z \sim 10)$ by the first stars (Pop III). Given the strong likelihood that Pop III stars rapidly enrich their environment with heavy elements and dust grains, such an assumption was in any case highly problematic.

Another major cosmological aspect of VHE $\gamma$-ray astronomy is an indirect Dark Matter search through the detection of annihilation radiation from the lowest-mass supersymmetric particles, called Neutralinos. Such weakly interacting massive particles are widely believed to constitute the Dark Matter in the Universe. From gravitational simulations they should be concentrated with a significant density increase in the central regions of Dark Matter halos, like the Galactic center (see below).

\section{Selected results}

\subsection{H.E.S.S. discovery of Pulsar B1259-630}

This $48 \mathrm{~ms}$ Pulsar is in a 3.4 yr-period highly excentric orbit around a Be star with blackbody temperature $T_{*}=23.000 \mathrm{~K}$. The particles from the Pulsar Wind are expected to be ultrarelativistic electrons and positrons, emitting Inverse Compton (IC) radiation in the stellar radiation field. Indeed the TeV-spectrum has been successfully predicted in the synchrotron/IC context (Kirk et al. (1999)). H.E.S.S. has observed the source for $\sim 50$ hrs in 2004 around perihelion; the flux was time-variable on the scales of days (Aharonian et al. (2005b)).

The Be star emits a strong disk-shaped wind that confines the Pulsar Wind Nebula into a "cometary" shape (where the analogy is meant morphologically rather than physically). The periastron lies behind the star (Fig. 1b). Unfortunately, moonlight did not allow observations during periastron itself. The data appear nevertheless to indicate a minimum of the VHE light curve near periastron (Fig. 2).

The IC losses, as measured by H.E.S.S. are about a factor of 10 weaker than the corresponding synchrotron losses, as inferred by INTEGRAL (Shaw et al. (2004)). This determines an effective magnetic field strength $B_{\text {eff }} \approx 1 \mathrm{G}$. In the expected Klein-Nishina regime the observed IC photon spectrum corresponds to a very hard differential electron spectrum with a power-law index $\alpha \geqslant 1.7 \pm 0.2$. It therefore cannot in turn come from a radiatively cooled electron population, and adiabatic losses must be dominant. This points to a rapidly and directionally expanding Pulsar Wind Nebula flow, resulting in a very interesting physical picture: The shocked Pulsar Wind is accelerated into a "cometary tail" flow, induced by the stellar wind. This flow is more strongly expanding outside the wind "disk" near periastron, where it is directed away from us. As a consequence its 


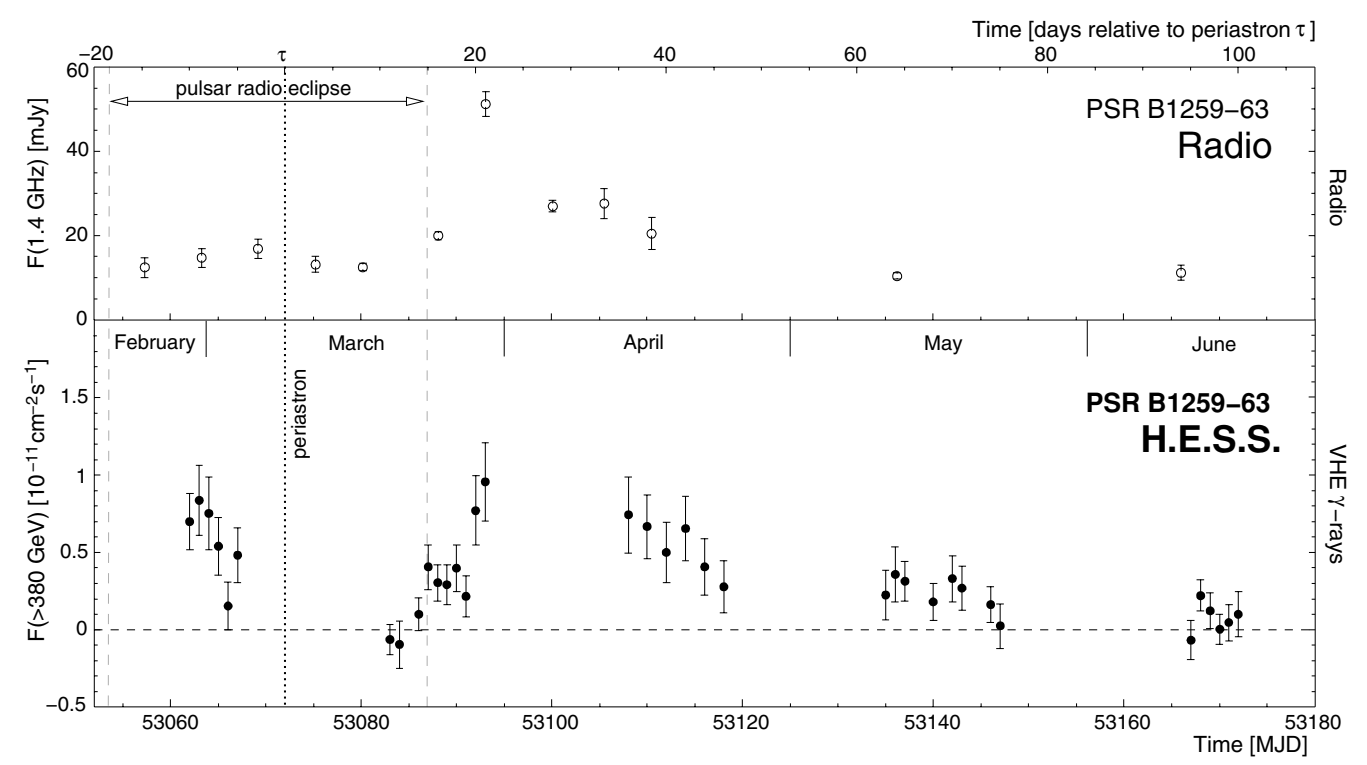

Figure 2. VHE $\gamma$-ray and unpulsed radio (Johnston et al. (2005)) light curves from PSR B1259-63 around its periastron passage (dotted vertical line).

emission is in addition diminished by Doppler dis-favoritism. Both effects together lead to a local minimum of the IC flux around periastron, despite the fact that the stellar radiation field peaks at this point.

\subsection{Galactic Center region}

The innermost region of our Galaxy has an abundance of nonthermal sources, many of them Supernova Remnants. H.E.S.S. has observed this region and has detected the neighborhood of Sgr A* with high significance (Aharonian et al. (2004a)). Two discrete sources were found, one around Sgr $\mathrm{A}^{*}$ itself and another one about $1^{\circ}$ away in the Galactic Disk (see Fig. 3a). This latter source is the Supernova Remnant (SNR) G0.9+0.1, dominated by a Pulsar Wind Nebula (Aharonian et al. (2005d)). Although the statistical angular resolution of the H.E.S.S. observation of the Sgr A* region is more than an order of magnitude better than the one achieved in other detections at $\mathrm{TeV}$ or $\mathrm{GeV}$ energies , the H.E.S.S. image center cannot yet be separated from $\mathrm{Sgr} \mathrm{A}^{*}$ itself within the present pointing accuracy of the system $(\sim 20 \operatorname{arcsec}$ in both coordinates). The $\gamma$-ray source is apparently point-like. Therefore other properties like the energy spectrum or a possible time variability become important criteria to determine whether the $\gamma$-ray emission comes from the central Black Hole and/or its immediate environment (the accretion flow or jets), or from a more extended source in the neighborhood. No time variability has been found until now. The form of the differential $\gamma$-ray spectrum $\propto E^{-2.3}$ (Fig. 3b) corresponds to a relatively hard power-law, and is therefore also consistent with freshly produced Cosmic Rays interacting with dense gas (with a hydrogen density $N_{H} \sim 10^{3} \mathrm{~cm}^{-3}$ ), or diffusive shock acceleration of particles still confined in the adjacent SNR Sgr A East. Theoretically there is even the possibility of a continuous transition between these two last radiation mechanisms.

The most exotic scenario would be dominant steady-state Neutralino/WIMP annihilation in the expected Dark Matter Halo centered on Sgr A*. Apart from the present difficulties to model the observed $\mathrm{TeV}$ spectrum within supersymmetric theories, the rest energy of the Neutralino must then be larger than the maximum energy up to which the 

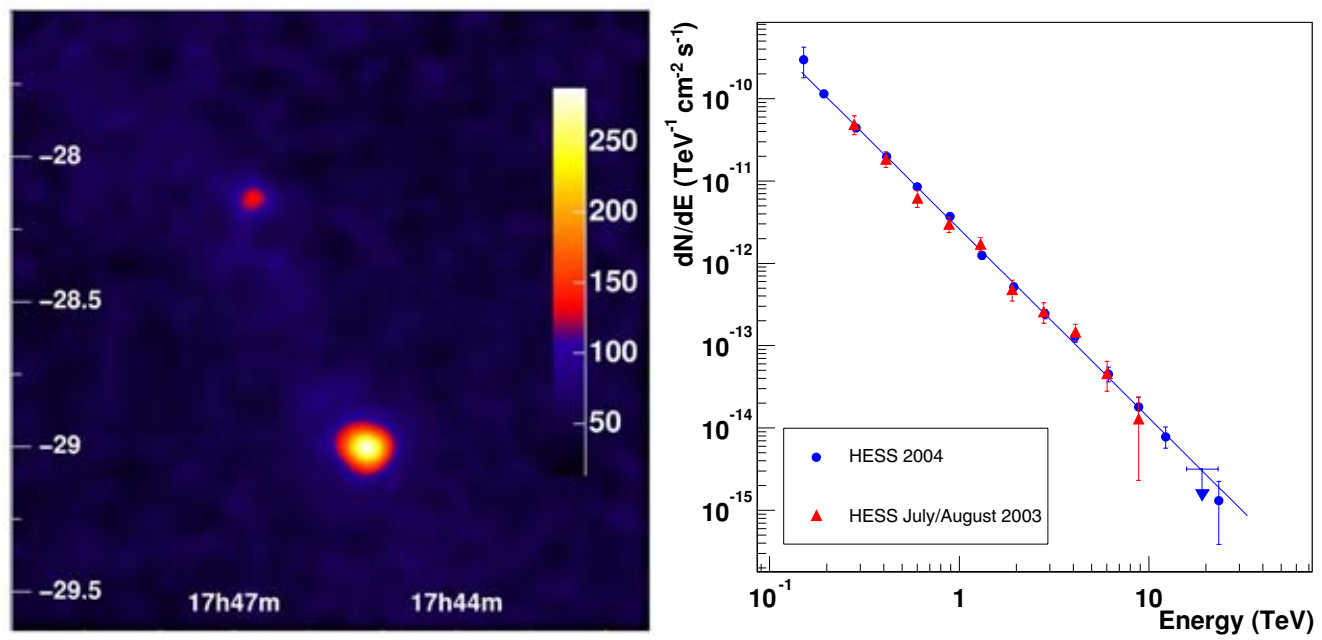

Figure 3. (Left:) H.E.S.S. VHE $\gamma$-ray image of the inner region of the Galaxy. The source on the lower right (Galactic Center region) is coincident within $1^{\prime}$ of Sgr A*. The source on the upper left is the Supernova Remnant G0.9+0.1 in the Galactic plane. (Right:) VHE $\gamma$-ray spectrum of the Galactic Center region as obtained by the H.E.S.S. array. The energy spectrum is a power law and extends beyond $20 \mathrm{TeV}$.

supposed annihilation spectrum extends. This corresponds to at least $20 \mathrm{TeV}$. Since the largest particle accelerator to exist in the foreseeable future, the $L H C$ in CERN, only reaches center-of-momentum energies of the order of a $\mathrm{TeV}$, this particle could not possibly be discovered in accelerator experiments. Thus, if the detected $\gamma$-ray flux from the Galactic center is to be ascribed to Dark Matter annihilations, then only astrophysics can tackle this question - a very interesting perspective.

\subsection{Supernova Remnants and Cosmic Ray origin}

While the theoretical aspects are essentially understood (e.g. Völk (2004) for a recent overview), there is a shortage of $\gamma$-ray detections. The EGRET experiment could not unequivocally establish the detection of a shell-type SNR in the GeV-range. Also the widely publicised $\mathrm{TeV} \gamma$-ray detection of SN 1006 by the CANGAROO experiment could not be confirmed by H.E.S.S. (Aharonian et al. (2005e)). The reason is in all probability the very low gas density $N_{H}<0.1 \mathrm{~cm}^{-3}$ (Ksenofontov et al. (2005)) in this object that is so bright in nonthermal X-rays (Koyama et al. (1995)). The second reason for the low $\mathrm{TeV}$ flux is the amplification of the magnetic field (Bell \& Lucek (2001), Berezhko et al. (2003a)) which depresses the IC emission - for given synchrotron emission - compared to that in a more typical $\mu \mathrm{G}$ interstellar field strength. Meanwhile also the CANGAROO collaboration has announced that it could not any more detect SN 1006 in stereoscopic re-observations (Mori (2005)).

There are only three VHE detections until now: RX J1713.7-3946 (Muraishi et al. (2000); Enomoto et al. (2002); Aharonian et al. (2004b)), Cas A (Aharonian et al. (2001)), and RX J0852.0-4622 (Katagiri et al. (2005); Aharonian et al. (2005f)).

Cas $\mathrm{A}$ is a thoroughly studied object in all wavelength ranges below $\gamma$-ray energies and thus amenable to a detailed application of nonlinear acceleration theory (Berezhko et al. (2003b)). In addition, the observations of narrow filamentary structures in nonthermal X-rays (assumed to be synchrotron radiation) by Vink \& Laming (2003) can be interpreted to imply an interior magnetic field strength of $500 \mu \mathrm{G}$ (Berezhko \& Völk (2004)), consistent with the theoretical analysis of the spatially integrated synchrotron spectrum. 

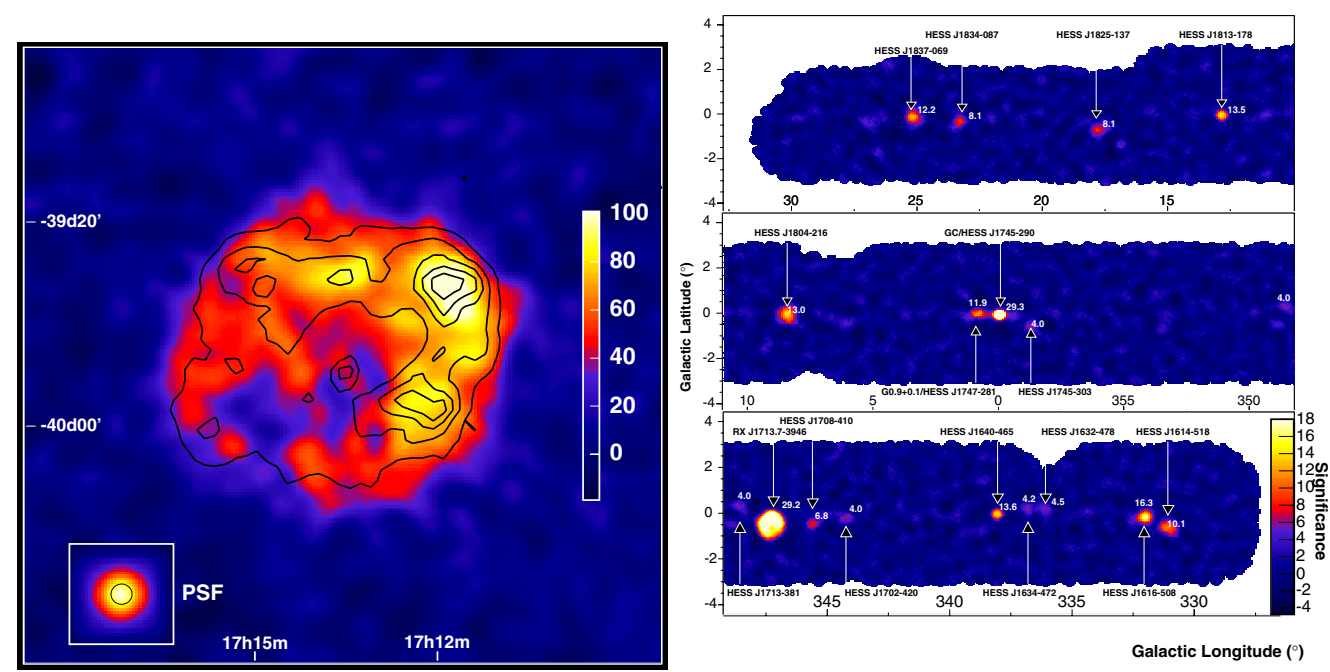

Figure 4. (Left:) Spatially resolved VHE $\gamma$-ray image of the SNR RX J1713.7-3936 as obtained by the H.E.S.S. array. The ASCA hard X-ray data (contour lines) are shown in addition. (Right:) H.E.S.S. scan of the Galactic Plane between $\pm 30^{\circ}$ in longitude and $\pm 3^{\circ}$ in latitude. The sources indicated are detected at a significance level greater than $4 \sigma$.

This strongly suggests Cas A as a source of nuclear Cosmic Rays, independent of all remaining uncertainties in the astronomical parameters.

The H.E.S.S. observations of RX J1713.7-3946 have for the first time resolved the morphology of an extended object in VHE gamma rays (Fig. 4a). The overall shell structure coincides closely in hard X-rays and gamma rays. Despite the complex structure this is unambiguous proof of the acceleration of charged particles to energies beyond $100 \mathrm{TeV}$. The most recent data show in addition that below about $10 \mathrm{TeV}$ the photon spectrum can be approximated by a power law in energy with an index close to 2.0. Such a spectrum is clearly consistent with diffusive shock acceleration. More detailed studies are in progress.

RX J0852.0-4622, also called "Vela Jr", was, like RX J1713.7-3946, first detected with the ROSAT telesope in X-rays (Aschenbach (1998)). The object has a radius of $\sim 1^{\circ}$, twice that of RX J1713.7-3946 and thus four times the radius of the full Moon. The $\mathrm{TeV} \gamma$-ray morphology correlates again very well with the $\mathrm{X}$-ray image, and the energy spectrum - not yet very precisely determined - can be fit by a power law with photon index 2.1. In this sense Vela Jr is similar to RX J1713.7-3946. Its apparently regular spherical shape and high $\gamma$-ray flux, comparable with that of the Crab Nebula, make it an ideal object for further studies.

\section{The H.E.S.S. survey of the Galactic plane}

In 2004 H.E.S.S. has completed the first 230 hour part of a VHE survey of the Galactic plane, covering $-30^{\circ}<\ell<30^{\circ}$ in longitude and $-3^{\circ}<b<3^{\circ}$ in latitude (Aharonian et al. $(2005 \mathrm{~g})$ ). The average flux sensitivity of the survey corresponds to $\sim 3$ percent of the Crab Nebula (Fig. 4a). Including recent re-observations of candidate sources from the initial survey fourteen previously unknown VHE sources have been found up to now (Aharonian et al. (2005h)). Most of the sources are still un-identified in other wavelength ranges. 
In this manner for the first time a $\mathrm{TeV}$ instrument has investigated not only source candidates that were well known from other wavelength ranges, but did a successful blind search. The search for counterparts in other wavelength ranges is ongoing. Most known counterpart candidates are young Pulsar Wind Nebulae or SNRs. The new sources have triggered a worldwide activity with satellite X-ray instruments like INTEGRAL, ASCA, $X M M$ and Astro-E2, and ground-based radio telesopes.

\section{Acknowledgements}

I would like to thank the members of the H.E.S.S. collaboration for many discussions on the observational results. I have learnt much from Felix Aharonian and Okkie de Jager about the physics of PSR B1259-63, even though I have of course the sole responsibility for the arguments in the text.

\section{References}

Aharonian, F.A. et al. (HEGRA Collaboration) 2001, A\& $A 370,112$

Aharonian, F.A. et al. (H.E.S.S. Collaboration) 2004a, A $6 A$ 425, L13

Aharonian, F.A. et al. (H.E.S.S. Collaboration) 2004b, Nature 432, 75

Aharonian, F.A. et al. (H.E.S.S. Collaboration) 2005a, submitted; arXiv:astro-ph/0508073

Aharonian, F.A. et al. (H.E.S.S. Collaboration) 2005b, $A \mathscr{G} A$, in press; arXiv:astro-ph/0506280

Aharonian, F.A. et al. (H.E.S.S. Collaboration) 2005c, $A \mathscr{G} A$ 439, 1013

Aharonian, F.A. et al. (H.E.S.S. Collaboration) 2005d, A\&A 432, L25

Aharonian, F.A. et al. (H.E.S.S. Collaboration) 2005e, $A \mathscr{E} A$ 437, 135

Aharonian, F.A. et al. (H.E.S.S. Collaboration) 2005f, $A \mathscr{E} A$, in press; arXiv:astro-ph/05/0538

Aharonian, F.A. et al. (H.E.S.S. Collaboration) 2005g, Science 307, 1938

Aharonian, F.A. et al. (H.E.S.S. Collaboration) 2005h, ApJ, to appear

Aschenbach, B. 1998, Nature 396, 141

Bell, A.R. \& Lucek, S.G. 2001, MNRAS 321, 433

Berezhko, E.G., Ksenofontov, L.T. \& Völk, H.J. 2003a, A $\& A$ 412, L11

Berezhko, E.G., Pühlhofer, G. \& Völk, H.J. 2003b, A\&\&A 400, 971

Berezhko, E.G. \& Völk, H.J. 2004, A\&̈A 419, L27

Cambresy, L., Reach, W.T., Beichman, C.A., et al. 2001, ApJ 555, 563

Enomoto, R., Tanimori, T., Naito, T. et al. 2002, Nature 416, 823

Johnston, S., Ball, L., Wang, N., et al. 2005, MNRAS 302, 277

Katagiri, H., Enomoto, R., Ksenofontov, L.T. et al. 2005, ApJ 619, L163

Kirk, J.G., Ball, L. \& Skjaeraasen, O. 1999, Astropart. Phys. 10, 31

Koyama, K., Petre, R., Gotthelf, E.V., et al. 1995, Nature 378, 255

Ksenofontov, L.T., Berezhko, E.G. \& Völk, H.J. 2005, A\& $A$, to appear; arXiv:astro-ph/0508318

Madau, P. \& Pozetti, L. 2000, Mon. Not. R. Astron. Soc. 312L, 9

Matsumoto, T., Matsuura, S., Murakami, H., et al. 2005, ApJ 626, 31

Mori, M. 2005, in: B. Degrange (ed.), Cherenkov2005, Proceedings Workshop "Towards a Network of Atmospheric Cherenkov Detectors VII" (Palaiseau, April 2005), to be published

Muraishi, H., Tanimori, T., Yanagita, S. et al. 2000, A\&A 354, L57

Shaw, S.E., Chernyakova, M., Rodriguez, J. et al. 2004, A\&A 426, L33

Vink, J. \& Laming, J. 2003, ApJ 548, 758

Völk, H.J. 2004, in: T. Kajita, Y. Asaoka, A. Kawachi, Y. Matsubara, \& M. Sasaki (eds.), Frontiers of Cosmic Ray Science, Proceedings 28th Int. Cosmic Ray Conf., Universal Academy Press, Inc. (Tokyo), vol. 8, p. 29

Weekes, T. C., Cawley, M. F., Fegan, D. J., et al. 1989, ApJ 342, 379 


\section{Discussion}

Chernyakova: Isn't the model of Kawacki et al. for PSRB 1259-63 better to explain the observed $\mathrm{TeV}$ emission, taking into account XMM observations of 2004 periastron passage, that are in favour of low Lorentz factor and IC mechanism for X-ray/Gamma-ray range?

VöLK: Speaker believes in IC mechanism for both X-ray and TeV-ray domain. [Answer recorded by questioner.]

Meurs: You showed a number of Galactic Plane new TeV sources that probably are SNRs. One the other hand you showed only a few TeV detected unknown SNRs. Is there any dichotomy between $\mathrm{TeV}$ and non TeV SNRs?

VÖLK: I do not think that there is a dichotomy. Theoretically the non-detection of SN 1006 is the result of a very low external gas density $\left(\mathrm{N}_{H}<0.1 \mathrm{~cm}^{-3}\right)$. Tycho's SNR is also a TeV upper limit only. Theoretically this UL is still a factor of a few above expectations. We need indeed some more TeV SNRs, but I see no problem for them being the source population of the Galactic Cosmic Rays. On the contrary, we are close to resolve this 100 yr old question.

UBERTINI: How do you assess the evidence of few SNRs seen in hard X-rays (in particular with the INTEGRAL survey) \& emitting TeV flux VS synchrotron/IC models.

VöLK: The maximum electron energy $\mathrm{p}_{\max }$ achieved by acceleration in the SNR blast wave is limited by synchrotron losses. Assuming the scattering mean free path $\sim$ gyro radius (Bohm limit), the synchrotron cut off frequency $\nu_{\max } \propto \mathrm{V}_{s}{ }^{2}$, where the shock velocity $\mathrm{V}_{s}$ decreases in time, especially after the initial sweep-up phase. Therefore $\nu_{\max }$ is in the hard X-ray range only for very young - and thus rare - SNRs (see Berezhko \& Völk, A\&A 427, 525 (2004), where this is discussed in detail). Thus we should not be surprised to see so few SNRs in hard X-rays. 